\title{
Conformal Array Pattern Synthesis Using a Hybrid GA/ABC Algorithm
}

\author{
C. Liu, L. H. Fan \\ Dept. of Communication and Engineering \\ Hefei University of Technology \\ Hefei, China
}

\begin{abstract}
A hybrid evolutionary algorithm is presented here to solve the problem of conformal array pattern synthesis. In order to overcome the disadvantage of standard genetic algorithm (GA) and artificial bee colony algorithm (ABC), a hybrid algorithm is introduced, which combines $G A$ and $A B C$ to take advantages of both methods. Crossover operator of GA is adopted to maintain the diversity of population. Multi-dimensional neighborhood search strategy of $A B C$ is introduced to improve the local search efficiency for conformal array pattern synthesis. Finally, the hybrid GA/ABC algorithm is used to optimize the weight vector of the circular conformal array. Experimental results show that the proposed method can achieve the desired pattern very well, and has a better performance than standard GA and ABC.
\end{abstract}

Keywords-conformal array; genetic algorithm (GA); artificial bee colony algorithm ( $A B C)$; hybrid GA/ABC algorithm; pattern synthesis

\section{INTRODUCTION}

Conformal arrays can be flexibility conformed to curved surface of platforms such as aerospace vehicles and ships [1]. This design lead to its advantages of excellent aerodynamic performance, low radar cross section (RCS), large surveillance coverage and volume saving. Consequently, there is an increasing demand in conformal arrays in many areas. However, the synthesis of conformal arrays that generate a desired radiation pattern is a highly nonlinear optimization problem. Those analytical methods for planar array are no more applicable.

Researches show that compare with the conventional approaches, the intelligent optimization algorithms such as the genetic algorithm (GA), particle swarm optimization (PSO) and ant colony optimization (ACO) can provide better performance and more flexible solutions for the pattern synthesis of conformal array [2]-[5]. Pattern synthesis is summarized as an optimization problem in the weight vector space. These intelligent optimization algorithms are used to search the optimal solution by defining suitable strategies and object function. Nevertheless, these algorithms have their own benefits and limitations in the array pattern synthesis. Therefore, some improved optimization algorithms [6][7] and hybrid algorithms [8]-[12] are developed to overcome their drawbacks.

Artificial bee colony (ABC) algorithm is proposed by Karaboga in 2005, which is simple in concept and requires very few initialization parameters to adjust [13]. It has properties of fast convergence speed, good quality of solutions and good robustness, etc [14][15]. But it also has the disadvantage of premature convergence, and is easy to fall into local optimum.
Genetic algorithm has good global search ability through its operation strategies, but has a slow convergence speed.

In this paper, a hybrid $\mathrm{GA} / \mathrm{ABC}$ algorithm is introduced for pattern synthesis of conformal arrays to take the advantages of both algorithms. Crossover operator of GA is adopted to maintain the diversity of population. Multi-dimensional neighborhood search strategy of $\mathrm{ABC}$ is introduced to improve the local search efficiency for conformal array pattern synthesis. The hybrid algorithm is used to search the optimal weight vector of conformal array to achieve a low side lobe level pattern with the constraint of main lobe shaping. At last, circular conformal array is used as an example to verify the effectiveness of proposed method. Simulation results illustrate the superior performance compare to $\mathrm{GA}$ and $\mathrm{ABC}$.

\section{PROBLEM FORMULATION}

Consider an arbitrary array consists of $N$ elements, the farfield radiation pattern $E(\theta)$ can be written as:

$$
E(\theta)=\sum_{i=1}^{N} w_{i} g_{i}(\theta) e^{j k\left(x_{i} \cos \theta+y_{i} \sin \theta\right)}=\mathbf{w}^{H} \mathbf{a}(\theta)
$$

where $g_{i}(\theta)$ represents directive element pattern of the $i$ th element, $(\cdot)^{H}$ denotes the conjugate transpose of the matrix, $\mathbf{a}(\theta)$ is the array steering vector and $\mathbf{w}$ is the excitation weight vector of array.

$$
\begin{aligned}
& \mathbf{a}(\theta)=\left[g_{1}(\theta) e^{j k\left(x_{1} \cos \theta+y_{1} \sin \theta\right)}, \cdots, g_{N}(\theta) e^{j k\left(x_{N} \cos \theta+y_{N} \sin \theta\right)}\right]^{T} \\
& \mathbf{w}=\left[\begin{array}{llll}
w_{1}, & w_{2}, & \cdots, & w_{N}
\end{array}\right]^{T}
\end{aligned}
$$

in which $x_{i}$ and $y_{i}$ are the positions of the $i$ th element, $k=2 \pi / \lambda$ is the phase constant and $\lambda$ is the free space wavelength.

In general, the problem of conformal array pattern synthesis is essentially to find the optimal excitation weight vector so that the array is capable to generate the desired beam pattern. Typically, the desired pattern is expected to remain within certain limits. Proper selected upper bound $U M(\theta)$ and lower bound $\operatorname{LM}(\theta)$ can be used to constraint the beam pattern, and then the fitness function can be defined as [16]:

$$
\text { Fit }=\sum_{l=1}^{L} \min \left(\left|E\left(\theta_{l}\right)\right|-\left|U M\left(\theta_{l}\right)\right|, 0\right)^{2}+\sum_{l=1}^{L} \min \left(\left|L M\left(\theta_{l}\right)\right|-\left|E\left(\theta_{l}\right)\right|, 0\right)^{2}
$$


where $E\left(\theta_{l}\right)$ is the realized pattern value from eqn. (1) evaluated at the sampling angle $\theta_{l}$. The pattern in eqn. (4) is calculated in decibel. Then, the optimal weight vector can be obtained by minimizing the above fitness function.

\section{THE HYBRID GA/ABC ALGORITHM}

\section{A. Artificial Bee Colony Algorithm}

Artificial bee colony algorithm simulates the foraging behaviors of honey bee swarm. The algorithm is capable of solving difficult multidimensional optimization problems through communication, transformation, and collaboration between the different roles of bees. The swarm intelligence search model contains two basic elements: food source and artificial bee colony, which include three kinds of roles: the employed bees, the onlookers, and the scouts.

A population of food sources is randomly generated initially with its upper and lower bound $\left(w_{i j}\right)_{\max }$ and $\left(w_{i j}\right)_{\min }$.

$$
w_{i j}=\left(w_{i j}\right)_{\min }+\operatorname{rand}[0,1]\left[\left(w_{i j}\right)_{\max }-\left(w_{i j}\right)_{\min }\right]
$$

Then, employed bees and onlookers search a neighboring food around the food source according to eqn. (6):

$$
v_{i j}=w_{i j}+R_{i j}\left(w_{i j}-w_{k j}\right)
$$

where $v_{i j}$ is new food source by searching around old food source $w_{i j}, w_{i j}$ and $w_{k j}$ denote the $i$ th and $k$ th food source, but $k$ must be different from $i$. $R_{i j}$ is a random number between $[-1,1] \cdot$

After all the employed bees complete their search process, they share the fitness value of the food sources with onlooker bees. Each onlooker bee randomly chooses a food source with a probability related to its fitness value provided by the employed bees. Since the problem needs to be solved is a minimization problem, tournament selection strategy is used as the selection mechanism.

The chosen source is still need to search its neighboring domain by eqn. (6). If the new source is better than the old one, replace it. And the roles of onlooker bee and employed bee will be exchanged. If the food source does not be replace for a predefined times, i.e., "limit", it will be abandoned and a new source will be generated by eqn. (5).

\section{B. Hybrid GA/ABC Algorithm}

In the above process we can find that the control parameter "limit" will affect its searching ability. A large value of it will strengthen its local searching capability but weaken the global searching capability. And a small value of it will have the opposite effect. In order to solve this conflict, crossover operation in GA is introduced here to intensify global searching ability and maintain the diversity of population. So that the hybrid algorithm will have a strong capability of local searching while avoiding premature convergence.

1) Crossover operation: For each generation, half of the solutions with smaller fitness values are selected to retain directly to the next generation. The remaining solutions of the population match each other randomly and perform crossover operation the same as GA.

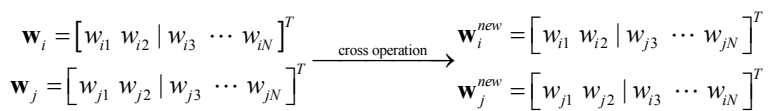

Compare with their parents, choose the better solutions with smaller fitness values and put them into the next generation.

2) Neighborhood Search Mechanism: Considering pattern synthesis problem of conformal array, each feasible solution of the excitation weight vector is a complex vector. Since the crossover operation is introduced to enhance the exploration capability, the neighborhood search strategy here needs to strengthen the exploitation capability and search efficiency. Considering these factors, neighborhood search strategy is replaced by the following equation:

$$
\mathbf{v}_{i}=\mathbf{w}_{i}+\sigma\left(\boldsymbol{\Delta}_{x i}+j \boldsymbol{\Delta}_{y i}\right)
$$

where $\boldsymbol{\Delta}_{x i}$ and $\boldsymbol{\Delta}_{y i}$ are $N$ dimensional vector randomly distributed on the range $[-1,1]$, and $\sigma$ is a small positive number to constraint the local search area.

In addition, scout bees behavior of original $\mathrm{ABC}$ algorithm is abandoned to further strengthen the exploitation capability.

3) Algorithm Summary: In order to make the procedure of the proposed algorithm clearer, the sequential steps of the proposed algorithm are given below.

Step 1: Define parameters such as population size $M$, maximum number of iteration $I_{\max }$ and feasible solution scope $\left[\left(w_{i j}\right)_{\min },\left(w_{i j}\right)_{\max }\right]$. Randomly generate the population of $M$ individuals $\mathbf{w}_{i}, i=1, \cdots, M$ according to eqn. (5).

Step 2: Neighborhood search is carried out for each individual by using eqn. (8) to find new solution around. Calculate its fitness value from the fitness function expressed in eqn. (4). Compare with the old one, and retain the individual with smaller fitness value of the two.

Step 3: Randomly select an individual by using tournament selection strategy, and execute the neighborhood search like step 2. Repeat this step $M$ times.

Step 4: Perform crossover operation of current population as described above.

Step 5: Record the optimal solution so far according to the fitness value of each individual.

Step 6: Repeat steps 2-5 until the maximum number of iterations is reached or a sufficiently good solution is found. 


\section{SimUlation ResUlts AND DisCUSSIONS}

The proposed hybrid algorithm is applicable to arbitrary arrays. To evaluate the capability of the proposed hybrid algorithm, it is simulated based on a 21-element uniform circular array as shown in figure 1 .

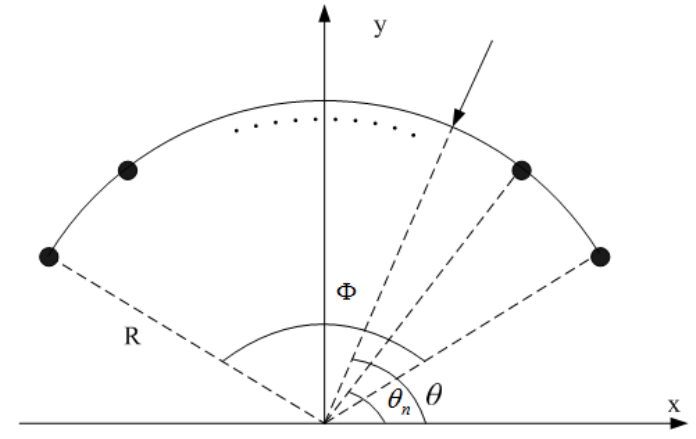

FIGURE I. N-ELEMENT UNIFORM CIRCULAR ARC ARRAY

The arc angle $\Phi$ is $150^{\circ}, \theta_{n}$ is the azimuth of $n$th element and the radius $R$ is $12 \lambda / \pi$. Therefore, the space between adjacent elements is half wavelength. In the following experiments, the function in eqn. (9) is chosen to be the element pattern.

$$
g_{n}(\theta)= \begin{cases}\cos \left(\theta-\theta_{n}\right) & \left|\left(\theta-\theta_{n}\right)\right| \leq \pi / 2 \\ 0 & \left|\left(\theta-\theta_{n}\right)\right|>\pi / 2\end{cases}
$$

The interested angle area is $\left[0,180^{\circ}\right]$ with its scan step $1^{\circ}$, and the number of sampling points is $L=181$ in eqn. (4).

In order to verify the superiority of the proposed hybrid algorithm denoted by $\mathrm{GA} / \mathrm{ABC}$, original $\mathrm{ABC}$ and $\mathrm{GA}$ based on real coding are selected to be the comparison algorithms. Since $\mathrm{GA} / \mathrm{ABC}$ and $\mathrm{ABC}$ is not sensitive to the population size, whereas GA has certain requirements on the size of population, the population size of three algorithms are set to be 30,30 and 100 , respectively. In addition, in order to make sure that all the algorithms is convergence, the maximum number of iterations is set to be $I_{\max }=3000$.

Figure 2(a) shows the finally obtained flattop beam pattern of different methods. The desired flattop beam pattern defined by the upper bound $U M(\theta)$ and lower bound $L M(\theta)$ can also be seen in figure 2 . The ripple in the main lobe need to be less than $2 \mathrm{~dB}$, the upper bound of the main lobe is $48^{\circ}$ and the lower is $40^{\circ}$ and desired side lobe level is less than $-30 \mathrm{~dB}$. From the result, we can see that all the three methods can obtain an acceptable pattern. But in the main lobe region, the fluctuation range of the proposed method is less than the other two algorithms. Figure 2(b) presents the convergence curves of three algorithms. It can be found that the proposed hybrid method have the fastest convergence rate.

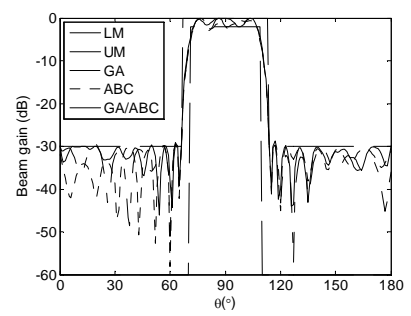

(a)

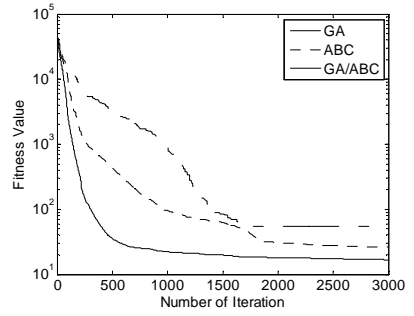

(b)
FIGURE II. (A) FLATTOP BEAM PATTERN FOR CIRCULAR CONFORMAL ARRAY (B) CONVERGENCE CURVES OF THREE ALGORITHMS

Figure 3(a) shows the results of cscsquare beam pattern for the three methods. The predefined upper and lower bound can also be seen on it. From the results, it can be found that the proposed hybrid method satisfied the constraint in both main lobe and side lobe area. Convergence curves shown in figure 3 (b) verify the better convergence rate of proposed algorithm again.

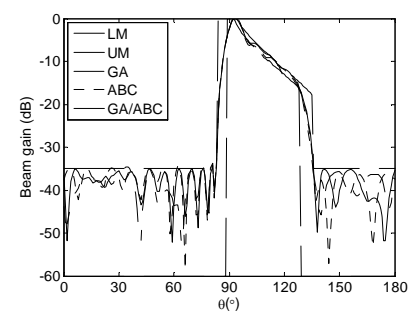

(a)

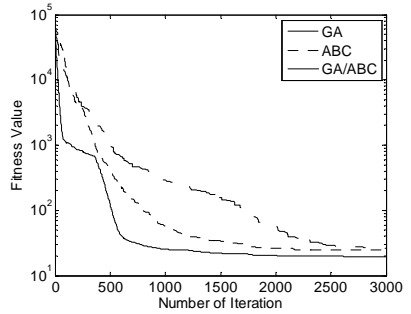

(b)
FIGURE III. (A) CSCSQUARE BEAM PATTERN FOR CIRCULAR CONFORMAL ARRAY (B) CONVERGENCE CURVES OF THREE ALGORITHMS

Table 1 demonstrates the finally fitness value of the above two simulations for the three methods, in which the proposed hybrid $\mathrm{GA} / \mathrm{ABC}$ algorithm has the best performance. From the results, it can be come to the conclusion that the proposed hybrid $\mathrm{GA} / \mathrm{ABC}$ algorithm improves the convergence rate with even better search accuracy compare with GA and ABC algorithm.

Table I. Fitness Value Comparison for Three Algorithms

\begin{tabular}{|c|l|l|l|}
\hline \multirow{2}{*}{ Algorithm } & \multirow{2}{*}{$\begin{array}{c}\text { Population } \\
\text { size }\end{array}$} & \multicolumn{2}{|c|}{ Best fitness value } \\
\cline { 3 - 4 } & 100 & 31.9254 & cscsquare \\
\hline GA & 100 & 26.0714 & 24.4546 \\
\hline ABC & 30 & 16.7051 & 19.6174 \\
\hline GA/ABC & 30 & \multicolumn{2}{|c}{} \\
\hline
\end{tabular}

\section{CONCLUSION}

In this paper, a hybrid $\mathrm{GA} / \mathrm{ABC}$ algorithm is presented to solve the pattern synthesis problem of conformal arrays. To achieve the desired pattern, the problem can be summarized as a minimum optimization problem by setting the upper and lower bound. Crossover operator of GA is introduced to improve the exploration capability and multidimensional neighborhood search strategy is introduced to improve the 
exploitation capability and search efficiency. Simulation results illustrate that the hybrid $\mathrm{GA} / \mathrm{ABC}$ algorithm has faster convergence rate and stronger searching ability compared with $\mathrm{GA}$ and $\mathrm{ABC}$ algorithm, and reveal that the proposed method can be an effective method to solve the solve the conformal array pattern synthesis.

\section{ACKNOWLEDGEMENT}

This work is supported by China Postdoctoral Science Foundation No. 20100480680 and the Natural Science Foundation of Anhui Province No. 1208085QF105.

\section{REFERENCE}

[1] L. Josefsson and P. Persson, Conformal Array Antenna Theory and Design. Piscataway. NJ: IEEE Press, 2006.

[2] C. M. Seong, M. Kang and C. S. Lee, Conformal array pattern synthesis on a curved surface with quadratic function using adaptive genetic algorithm. IEEE Asia-Pacific Microwave Conference Proceedings, pp.167-169, 2013.

[3] F. Zhao, X. Ke and T. T. Liu, Adaptive dynamic meta particle swarm optimization algorithm and application in 3D power pattern synthesis for conformal arrays. IEEE International Conference on Microwave and Millimeter Wave Technology, vol.5, pp.1-5, 2012.

[4] M. Lanza, J. R. Pérez and J. Basterrechea, Particle Swarm Optimization applied to planar arrays synthesis using subarrays. IEEE Proceedings of the Fourth European Conference on Antennas and Propagation, vol.5, pp.1-5, 2010.

[5] O. Quevedo-Teruel and E. Rajo-Iglesias, Ant Colony Optimization for Array Synthesis. IEEE International Symposium on Antennas and Propagation Society, pp.3301-3304, 2006.

[6] L. Cen, Z. L. Yu and W. Ser, Linear aperiodic array synthesis using an improved genetic algorithm. IEEE Transactions on Antennas and Propagation, vol.60, no.2, pp. 895-902, 2012.

[7] W. T. Li, X. W. Shi and Y. Q. Hei, An improved particle swarm optimization algorithm for pattern synthesis of phased arrays. Progress In Electromagnetics Research, vol.82, pp. 319-332, 2008.

[8] W. T. Li, X. W. Shi and Y. Q. Hei, A hybrid optimization algorithm and its application for conformal array pattern synthesis. IEEE Transactions on Antennas and Propagation, vol.58, no.10, pp. 3401-3406, 2010.

[9] B. R. Karimzadeh, K. Forooraghi and S. Chamaani, Conformal Array Pattern Synthesis Using a Hybrid WARP/2LB-MOPSO Algorithm. International Journal of Antennas and Propagation, pp.1-7, 2012.

[10] Y. Y. Bai, S. Xiao and C. Liu, A hybrid IWO/PSO algorithm for pattern synthesis of conformal phased arrays. IEEE Transactions on Antennas and Propagation, vol.64, no.4, pp. 2328-2332, 2013.

[11] W. T. Li, X. W. Shi and Y. Q. Hei, A hybrid optimization algorithm and its application for conformal array pattern synthesis. IEEE Transactions on Antennas and Propagation, vol.58, no.10, pp. 3401-3406, 2010.

[12] J. Yang, W. Li and X. Shi, A Hybrid ABC-DE Algorithm and Its Application for Time-Modulated Arrays Pattern Synthesis. IEEE Transactions on Antennas and Propagation, vol. 61, no. 11, pp. 54855495, Nov. 2013.

[13] D. Karaboga, An idea based on honey bee swarm for numerical optimization. Technical report-tr06, Erciyes University, engineering faculty, computer engineering department, 2005.

[14] B. Wang and L. Wang., A novel artificial bee colony algorithm for numerical function optimization. IEEE Fourth International Conference on Computational and Information Sciences, pp. 172-175, 2012.

[15] G. Zhu and S. Kwong, Gbest-guided artificial bee colony algorithm for numerical function optimization. Applied Mathematics and Computation, vol. 217, no. 7, pp. 3166-3173, 2010.

[16] J. R. P. Lopez and J. B. Verdeja, Synthesis of linear arrays using particle swarm optimisation. IEEE First European Conference on Antennas and Propagation, pp. 1-6, 2006. 